\title{
Dual Encoding for Abstractive Text Summarization
}

\author{
Kaichun Yao, Libo Zhang, Dawei Du, Tiejian Luo, Lili Tao and Yanjun Wu
}

\begin{abstract}
Recurrent Neural Network (RNN) based sequenceto-sequence attentional models have proven effective in abstractive text summarization. In this paper, we model abstractive text summarization using a dual encoding model. Different from the previous works only using a single encoder, the proposed method employs a dual encoder including the primary and the secondary encoders. Specifically, the primary encoder conducts coarse encoding in a regular way, while the secondary encoder models the importance of words and generates more fine encoding based on the input raw text and the previously generated output text summarization. The two level encodings are combined and fed into the decoder to generate more diverse summary that can decrease repetition phenomenon for long sequence generation. The experimental results on two challenging datasets (i.e., CNN/DailyMail and DUC 2004) demonstrate that our dual encoding model performs against existing methods.
\end{abstract}

Index Terms-recurrent neural network, abstractive text summarization, dual encoding, primary encoder, secondary encoder.

\section{INTRODUCTION}

$\mathbf{T}$ EXT summarization aims to generate short, accurate and informative summary from larger text documents. It is widely applied in natural language understanding and information retrieval, etc. Summarization techniques are mainly grouped into extractive and abstractive approaches. Extractive methods construct a summary by extracting salient words, phrases or sentences from the source text. Abstractive methods produce a summary similar to a human-written abstract by concisely paraphrasing the source content. That is, the former ensures the grammatical and semantic correctness of the generated summaries, while the latter creates more diverse and novel content. In this work, we focus on abstractive text summarization.

Recently, neural networks are widely leveraged in many natural language processing tasks because of promising performance [1]. Specifically, the neural networks based encoderdecoder models are used in the sequence-to-sequence tasks such as neural machine translation [2], [3], speech recognition [4], [5], image captioning [6], conversational system [7] and text summarization [8], [9], [10], [11]. Within text summarization tasks, the encoder reads the whole input sequence and generates a fixed dimensional feature vector, and then the decoder uses the feature representation to produce desired output sequence. For example, the summarization model employs a Convolutional Neural Network (CNN) as an encoder and a feed-forward neural network language model as a decoder [8].

Kaichun Yao, Dawei Du and Tiejian Luo are with the University of Chinese Academy of Sciences. Libo Zhang and Yanjun Wu are with Institute of Software Chinese Academy of Sciences and State Key Laboratory of Computer Science. Lili Tao is with University of the West of England.

Corresponding author: Libo Zhang(libo@iscas.ac.cn). This work is supported by National Natural Science Foundation of China (Grant No. 61807033).
An extension to this framework is to add attention mechanism by considering context cues in hidden states of the encoder, which facilitates to decode the target sequence [12].

Although the above encoder-decoder models are promising, some problems still remain. In these works, a decoder uses a fixed target vocabulary to output the corresponding probability distribution at each timestep. This may lead to the incapable to handle rare or Out-Of-Vocabulary (OOV) words. Increasing the size of the target vocabulary could alleviate this problem, but this increases the computational complexity in decoding as a softmax function needs to calculate over all possible words. This can be improved by applying a copy mechanism that dynamically copies the words from the input sequence when decoding without enlarging the size of the vocabulary [10], [13], [14], [15], [16].

Another problem with encoder-decoder models is that they often generate unnatural summaries consisting of repeated phrases, especially evident for long text summarization generation. On a long sentence summarization dataset (e.g., $\mathrm{CNN} /$ DailyMail dataset containing multi-sentence summaries of up to 56 tokens on average), a coverage mechanism is used to avoid the repetition problem. It records past attentional weights in the decoder and dampens the decoder from attending to the same parts of the input text when decoding in future [13]. Different from this mechanism, an intra-attention mechanism takes the attention at the decoder into account, which is prominently effective for eliminating repetition [17]. However, they consider little about the relations between the input tokens in the encoder and the already generated words by decoder.

To solve these problems, we propose a novel Dual Encoding for Abstractive Text Summarization (DEATS) that extends the existing sequence-to-sequence framework. Specifically, our dual encoding model consists of a primary encoder, a secondary encoder and a decoder. It conducts the primary encoder and the decoder as the standard attentional encoderdecoder model. The secondary encoder is based on the input and the previously produced output, and generates a new context vector as an additional input of the decoder. The context vector makes the decoder obtain more meaningful information and generate better output. Besides, we conduct a multi-step decoding operation in the decoder, and model the decoded content at each stage as a semantic feature vector. This makes the decoder "remember" the content produced in the earlier time-steps in order to avoid the repetition. The main contributions of this paper are summarized as follows:

- We propose a dual encoding mechanism to extend the traditional sequence-to-sequence model to make full use of the document text information by adding an additional encoder. 
- We consider the importance of words in the input to make the secondary encoder re-weigh "remembered" and "forgotten" parts in the input sequence.

- We introduce an enhanced repetition avoidance mechanism. It combines an existing coverage mechanism and the previously decoded content produced in the earlier time-steps to improve the repetition problem in sequence generation tasks.

- We conduct experiments on two challenging datasets (i.e., the CNN/DailyMail dataset and the DUC 2004 dataset), which shows that our dual encoding model outperforms existing models.

The remainder of this paper is organized as follows. Related work on text summarization is reviewed in Section [I The proposed approach is presented in Section III] Experimental evaluations and discussions are given in Section IV and Section $\mathrm{V}$. respectively. Concluding remarks are given in Section VI

\section{RELATED WORK}

\section{A. Text Summarization}

A large majority of work in the past few years has been focused on extractive summarization [18], [19], [20], [21], [22], [23], [24], [25], [26], where a summary consists of key words or sentences from the source text. Different from extractive methods copying units from the source article directly, abstractive summarization uses the readable language for human to summarize the key information of the original text. Therefore, abstractive approaches can produce much more diverse and richer summaries. Abstractive summarization task has been standardized by the DUC2003 and DUC2004 competitions [27]. Hence, there emerge a series of notable methods without neural networks on this task, e.g., the best performer TOPIARY system [28].

Recently, the emergence of the generative neural models [12] for text has inspired new work in abstractive summarization. A neural network model uses a convolutional encoder to encode the source and attentional feed-forward network to produce a summary [8]. It achieves the state-of-the-art results on the DUC-2004 1 and Gigaword datasets. An extension of this work uses a similar encoder but replaces the decoder with a Recurrent Neural Network (RNN) [9], and achieves better performance on the both above datasets. Apart from English text summarization, a large dataset for Chinese short text summarisation is introduced. The context is used as input of the decoder which is computed as a sum of all hidden states from the encoder [29].

\section{B. Rare or OOV Words Problem}

Rare and OOV words prevent models from learning representations for new words during training. This may result in a poor readability for the generated summaries. Although RNN based encoder-decoder models with attention have shown good performance on many datasets, it is challenging to model rare or OOV words effectively. To handle this problem, a pointer

${ }^{1}$ http://duc.nist.gov/duc2004/tasks.html mechanism is proposed to use a new decoder network to point back to OOV words and phrases in the input text and copy them into the output [30]. Furthermore, an approach combining the pointer mechanism and the original word generation layer in the decoder considers either of them at each decoding step [13], [27]. Different from the work in [13], [27], the model with encoder-decoder structure integrates the copying mechanism with word generation in the decoder [15]. Another copying mechanism derives the representations of OOV words from their corresponding context in the input text [16].

\section{Repetition Problem}

A common issue of neural networks based encoder-decoder models is that they tend to generate repetitive and incoherent phrases in longer summaries. To avoid this, a coverage mechanism eliminates the repetition by discouraging it from attending to the same part in the input sequence when decoding [14]. The method is adapted from statistical machine translation tasks [31], [32]. A distraction mechanism can be incorporated into the neural networks based summarization model [33]. All these approaches are devoted to the different forms of information encoding and acquisition at the encoder. On the contrary, the decoded information at the decoder can also be used to avoid the repetition [17]. Our dual encoding model is similar to [16] at the encoder, but conducts a different secondary encoding every several decoding steps. At the same time, at the decoder, the decoded content is modelled as a feature representation and then the secondary encoder uses it to fulfil a secondary encoding.

The repetition problem happens more often in long sequence generation tasks. However, researchers pay little attention to large-scale datasets for summarization of longer text. In [10], the RNN based encoder-decoder model with hierarchical attention is proposed for abstractive summarization task and evaluated on the CNN/DailyMail dataset [34]. Later, another hierarchical RNN model is developed and achieves significantly better abstractive result with respect to the ROUGE metric [35]. Our dual encoding model is mainly designed for long sequence generation tasks, therefore we also use the $\mathrm{CNN} /$ DailyMail dataset to evaluate the proposed method.

\section{Dual Encoding Model}

Abstractive text summarization can be formulated as a generation task that a "output sequence" is generated from a "input sequence". The input is a source text sequence $X=$ $\left[x_{1}, x_{2}, \cdots, x_{j}, \cdots, x_{m}\right]$, where $j$ and $m$ are the index and the number of the words in source text, respectively. The output is a shorter summary sequence $Y=\left[y_{1}, y_{2}, \cdots, y_{i}, \cdots, y_{n}\right]$ of that text, where $i$ and $n$ are the index and the number of the words in summary text, respectively.

In this section, we describe the dual encoding model in detail. As depicted in Fig. 1, our dual encoding model consists of a primary encoder, a secondary encoder and a decoder equipped with an attention mechanism:

- The primary encoder calculates the semantic vectors for each word in input sequence. 


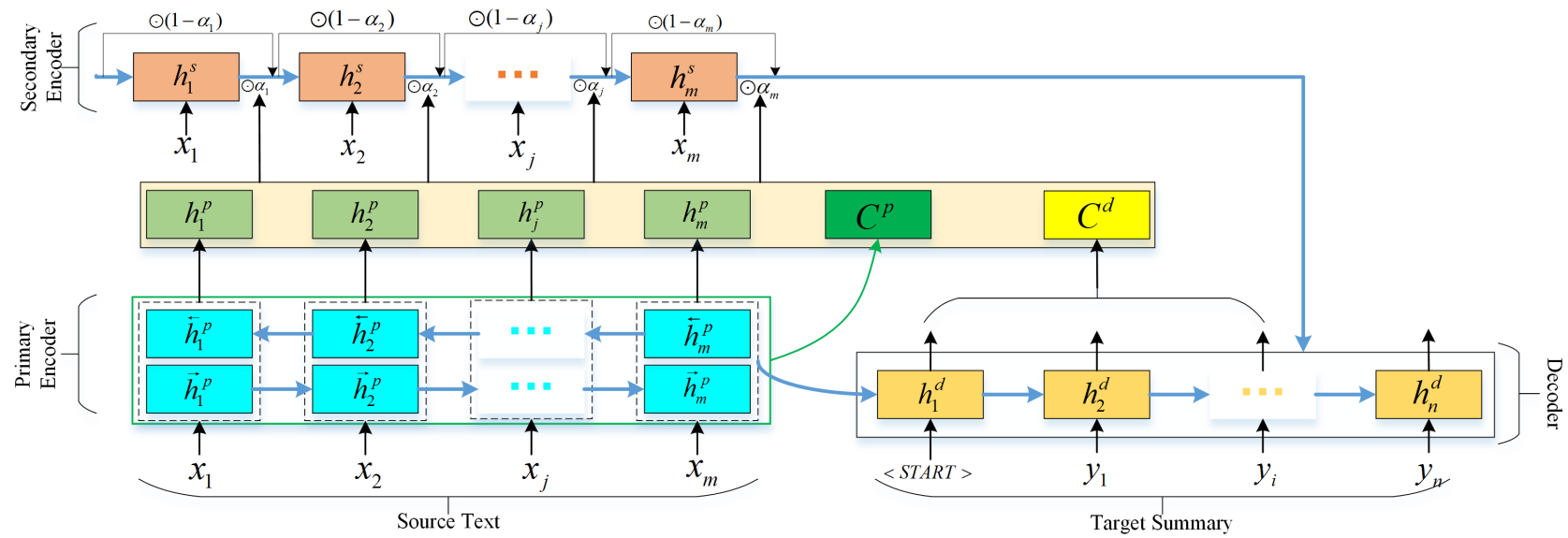

Fig. 1. Overview of our dual encoding model.

- The secondary encoder first calculates the importance weight for each word in input sequence and then recalculates the corresponding semantic vectors.

- The decoder with attention mechanism decodes by stages and generates a partial fixed-length output sequence at each stage.

Notably, all the above three modules employ the Gated Recurrent Unit (GRU) [36].

For each iteration, the primary encoder first reads each word in the input sequence, and produces the corresponding hidden representation (i.e., $h_{j}^{p}$ in Fig. 1) and the content representation (i.e., $C^{p}$ in Fig. 11) for the whole source text.

Secondly, the decoder generates the partial fixed-length sequence for every $K$ decoding steps which is modelled as the current decoded content representation (i.e., $C^{d}$ in Fig. 1), where $K$ is defined in Eq. (9). Based on the above representations $\left(h_{j}^{p}, C^{p}\right.$ and $\left.C^{d}\right)$, the importance weight (i.e., $\alpha_{j}$ in Fig. 1) is calculated with Eq. (4).

Thirdly, the secondary encoder fulfils more fine encoding on the input sequence for every $K$ decoding steps. Specially, we put the $\alpha_{j}$ on the secondary encoder in the form of skipconnections. Then, the secondary encoding is conducted on the source text followed in Eq. (5). It is worth mentioning that the new encoding generates a new semantic context vector (i.e., $h_{m}^{s}$ in Fig. 1) to facilitate the decoder to output more accurate target sequence. When the decoder finishes decoding the next fixed-length ( $K$ decoding steps) subsequence, the secondary encoder conducts the secondary encoding once again.

In our dual encoding model, the secondary encoder conducts an encoding operation based on the input and already output at each stage. Therefore, It is of great significance for the quality of the previously texts generated by the decoder. The better previous output would significantly facilitate the latter text generation. We use the pointer mechanism and the coverage mechanism to guarantee the decoder to obtain a better partial output. A combination of the dual encoding mechanism, the pointer mechanism and the coverage mechanism could make them benefit from each other.

The details of the primary and the secondary encoder are described in Section III-A and Section III-B, respectively.
Different from the general decoder, our decoder conducts the decoding operation by stages, which is described in Section III-C. We use the pointer mechanism to handle rare or OOV words and a enhanced repetition avoidance mechanism to discourage the repetition problem, which are given in Section III-D and Section III-E, respectively. Besides, the training process for our dual encoding model is given in Algorithm 1

\section{A. Primary Encoder}

RNN has achieved promising performance in sequence processing tasks, especially in handling a variable-length sequence [37]. Moreover, RNN with the gating units is more easily trained than vanilla RNN with better peformance in many tasks [38]. Therefore, we employ the primary encoder to generate coarse encoding using a GRU based RNN [38]. A GRU can adaptively capture dependencies of different time scales, which is defined as the following equations.

$$
\left\{\begin{array}{l}
u_{t}=\sigma\left(W_{u}\left[x_{t}, h_{t-1}\right]\right), \\
r_{t}=\sigma\left(W_{r}\left[x_{t}, h_{t-1}\right]\right), \\
\left.h_{t}^{\prime}=\tanh \left(W_{h}\left[x_{t}, r_{t} \odot h_{t-1}\right)\right]\right), \\
h_{t}=\left(1-u_{t}\right) \odot h_{t-1}+u_{t} \odot h_{t}^{\prime},
\end{array}\right.
$$

where $W_{u}, W_{r}$ and $W_{h}$ are parameter matrices. $x_{t}$ and $h_{t}$ indicate the corresponding input embedding vector and the hidden state vector at the time step $t$, and $\odot$ is an elementwise multiplication operator.

The purpose of the primary encoder is to construct the feature representation of the input sentence. Here we employ a bidirectional GRU (Bi-GRU) as the recurrent unit of the primary encoder, as shown in the bottom-left of Fig. 11 The Bi-GRU consists of a forward and a backward GRU. Given a sequence of the input word embeddings (i.e., $\left(x_{1}, x_{2}, x_{j}, x_{m}\right)$ in Fig. 11, the forward GRU computes hidden state representations $\left(\vec{h}_{1}^{p}, \vec{h}_{2}^{p}, \cdots, \vec{h}_{j}^{p}, \cdots \vec{h}_{m}^{p}\right)$ at each word position sequentially according to the current word embedding and the previous hidden state. The backward GRU generates hidden state representations $\left(\overleftarrow{h}_{1}^{p}, \leftarrow_{h}^{p}, \cdots, \overleftarrow{h}_{j}^{p}, \cdots, \leftarrow_{h}^{p}\right)$ for each word 


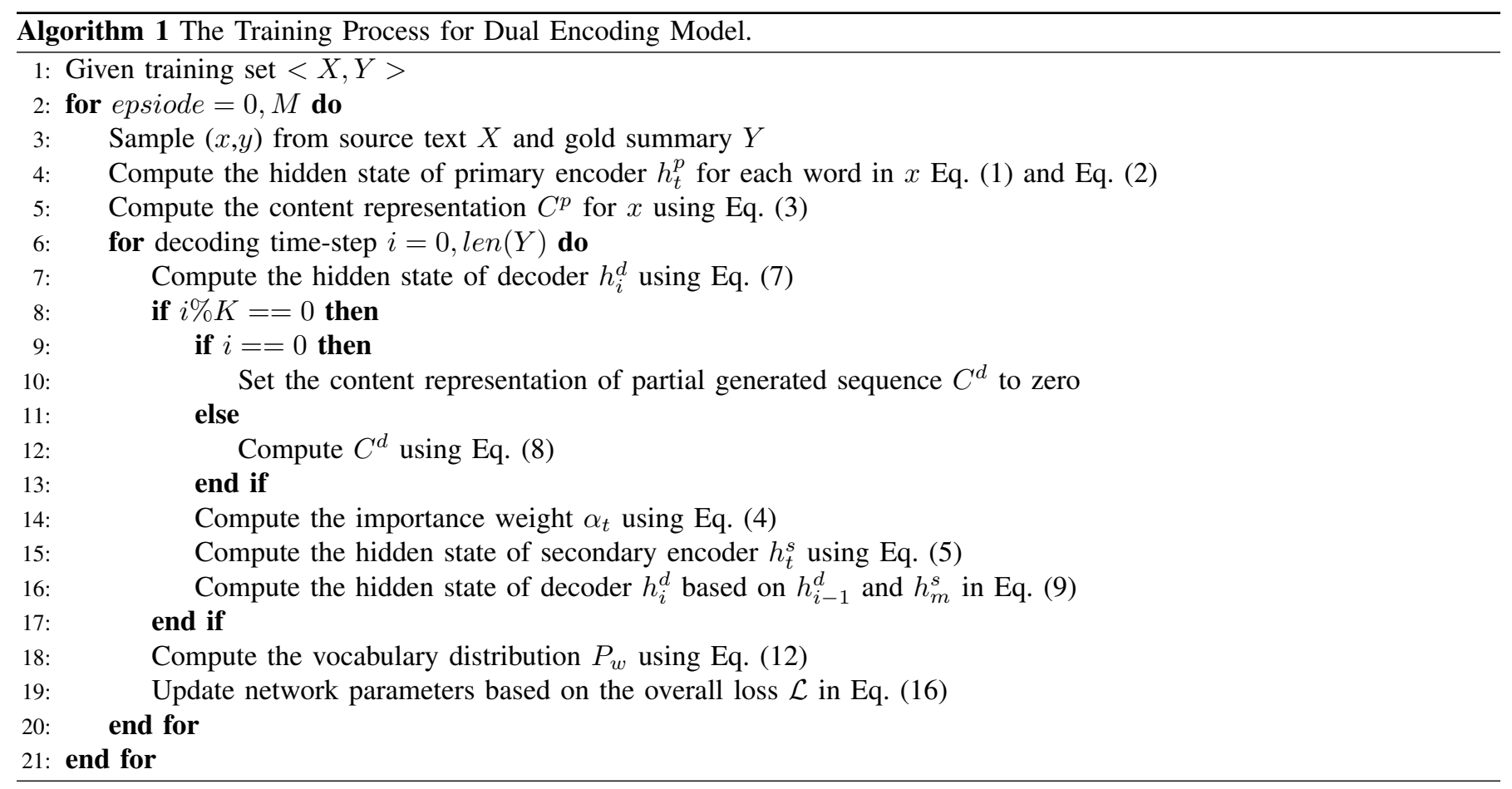

in reversed sequence (i.e., from the last word to the first). The two kinds of hidden states are defined as

$$
\left\{\begin{array}{l}
\vec{h}_{t}^{p}=\operatorname{GRU}^{p}\left(x_{t}, \vec{h}_{t-1}^{p}\right), \\
\overleftarrow{h}_{t}^{p}=\operatorname{GRU}^{p}\left(x_{t}, \stackrel{\leftarrow}{h}_{t-1}^{p}\right) .
\end{array}\right.
$$

We set the initial states of the Bi-GRU to zero vectors, i.e., $\vec{h}_{1}^{p}=\mathbf{0}$ and $\overleftarrow{h}_{m}^{p}=\mathbf{0}$. After the input sequence is read by the primary encoder, each word in the sequence can be represented as a concatenated hidden state of forward GRU and backward GRU, denoted as $h_{t}^{p}=\left[\vec{h}_{t}^{p}, \stackrel{\leftarrow}{h}_{t}^{p}\right]\left(\right.$ i.e. $, h_{1}^{p}, h_{2}^{p}, h_{j}^{p}, h_{m}^{p}$ in Fig 1 ). Then, we can model the representation of the whole input text sequence as a non-linear transformation of the average pooling of the concatenated hidden states of Bi-GRU. The representation $C^{p}$ is calculated as

$$
C^{p}=\tanh \left(W_{p} \frac{1}{N} \sum_{t=1}^{N} h_{t}^{p}+b_{p}\right),
$$

where $W_{p}$ and $b_{p}$ are parameters, and $N$ represents the length of the input sequence.

\section{B. Secondary Encoder}

The secondary encoder is depicted in the top of Fig. 1 As discussed above, the primary encoder reads the input sequence only once to create the hidden state representations. It computes the context with attention mechanism at each decoding time step adaptively. Different from the primary encoder, the secondary encoder is built with unidirectional GRU RNN, and reads the input sequence every $K$ decoding steps according to the decoded information at each stage. At the same time, the importance weight $\alpha_{t}$ is computed based on the feature representation of each word $h_{t}^{p}$ in the input sequence, the content of entire input text sequence $C^{p}$ and the content representation of output sequence $C^{d}$ generated by decoder at the current stage. We have

$$
\begin{aligned}
\alpha_{t}= & \sigma\left(W_{2}\left(\tanh \left(W_{1}\left[h_{t}^{p}, C^{p}, C^{d}\right]+b_{1}\right)\right)+h_{t}^{p T} W_{s} C^{p}\right. \\
& +h_{t}^{p T} W_{s} C^{d}-C^{p T} W_{r} C^{d}+b_{2},
\end{aligned}
$$

where $W_{1}, W_{2}, W_{s}, W_{r}, b_{1}$ and $b_{2}$ are the learning parameters. The importance weight $\alpha_{t}$ signifies how much attention should be paid to the current input word $x_{t}$. For the summarization task, the saliency between every word and the entire content of source text is modelled as $h_{t}^{p T} W_{s} C^{p}$ and $h_{t}^{p T} W_{s} C^{d}$ in Eq. (4). The redundancy between the content of source text and the decoded content in current stage is modelled as $C^{p^{T}} W_{r} C^{d}$ in Eq. (4). Finally, $\alpha_{t}$ is computed for each word in the input sequence based on the information itself, its saliency and the redundancy.

As shown in Fig. 11 we put the importance weight $\alpha_{t}$ on the skip-connections to bias the two information flows. That is, if the current input word $x_{t}$ has a very small weight $\alpha_{t}$, then the hidden state $h_{t}^{s}$ encoded by the secondary encoder will take the majority of information directly from the previous hidden state $h_{t-1}^{s}$, neglecting the effect of the current word. If $\alpha_{t}$ approximates to 1 , it is similar to a standard GRU, which is only influenced from the current word. Thus the secondary encoder has the following update rule:

$$
h_{t}^{s}=\left(1-\alpha_{t}\right) \odot h_{t-1}^{s}+\alpha_{t} \odot \mathrm{GRU}^{s}\left(x_{t}, h_{t-1}^{s}\right) .
$$

Notably, the final hidden state $h_{m}^{s}$ is the complementary information to help decoder generate target summary. Thus both the secondary encoder and the primary encoder together complete our dual encoding process.

\section{Decoding by Stages}

As shown in the right of Fig. 1, we also use GRU as the decoder to generate the output summary. The decoder 
and primary encoder constitute a basic sequence-to-sequence model. Moreover, some advanced techniques, such as attention mechanism [12], copy mechanism [15], [16], pointer-generator network [10], [13], [14] and coverage mechanism [14], [31] can be applied in the basic sequence-to-sequence model to achieve better performance. In the sequence generation task, the secondary encoder is used as a complementary and independent encoder to improve the performance of our basic model. In this work, we use a decoder with attention mechanism to compute the context vector according to the hidden states $\left(h_{1}^{p}, h_{2}^{p}, \cdots, h_{j}^{p}, \cdots, h_{m}^{p}\right)$ of the primary encoder. The context vector $c_{i}$ is computed as a weighted sum of these hidden states as

$$
c_{i}=\sum_{j=1}^{n} a_{i j} h_{j}^{p},
$$

where the weight $a_{i j}$ of each hidden state $h_{j}^{p}$ is computed by

$$
\left\{\begin{array}{l}
a_{i j}=\frac{\exp \left(e_{i j}\right)}{\sum_{k=1}^{n} \exp \left(e_{i k}\right)}, \\
e_{i j}=v_{a}^{T} \tanh \left(W_{a} h_{i-1}^{d}+U_{a} h_{j}^{p}\right), \\
h_{i}^{d}=G R U^{d}\left(y_{i}, h_{i-1}^{d}\right) .
\end{array}\right.
$$

Score $e_{i j}$ represents how well the inputs around position $j$ match the output at position $i$, and $h_{i}^{d}$ is the hidden state generated by the decoder that is based on its last hidden state $h_{i-1}^{d}$ and the $i$-th target $y_{i}$ in the output sequence.

Our dual encoding model does not decode the whole output sequence at one time but decodes the partial fixed-length sequence by stages. We decode the partial sequence for the fixed length $K$ at each stage and model the whole decoded sequence as

$$
C^{d}=\tanh \left(W_{d} \frac{1}{L} \sum_{i=1}^{L} h_{i}^{p}+b_{d}\right),
$$

where $W_{d}$ and $b_{d}$ are parameters, and $L$ denotes the length of the current decoded sequence. $C^{d}$ is the current content produced by the decoder, which is used to adjust the attention weight of the secondary encoder to each word in the input sequence, and we set $C^{d}$ to a zero vector at the beginning of decoding. After every fixed-length decoding, the secondary encoder generate a new final state $h_{m}^{s}$, and our decoder is rewritten as follows.

$$
h_{i}^{d}= \begin{cases}\operatorname{GRU}^{d}\left(y_{i},\left[h_{i-1}^{d}, h_{m}^{s}\right]\right) & \text { if } L \% K==0 \\ \operatorname{GRU}^{d}\left(y_{i}, h_{i-1}^{d}\right) & \text { otherwise. }\end{cases}
$$

The initial state of the decoder is set to the final state of the primary encoder, namely $h_{0}^{d}=h_{m}^{p}$. We compute the decoded content and the secondary encoding at every $K$ decoding steps. Then, we concatenate the current context vector $c_{i}$ acquired from the primary encoder and the decoder hidden state $h_{i}^{d}$, and feed through one linear layer to produce the vocabulary distribution as

$$
P_{v}=P\left(y_{i} \mid y_{1}, \cdots, y_{i-1} ; x\right)=\operatorname{softmax}\left(W_{v}\left[h_{i}^{d}, c_{i}\right]+b_{v}\right),
$$

where $P\left(y_{i} \mid y_{1}, \cdots, y_{i-1} ; x\right)$ is the conditional probability distribution for the target word $y_{i}$ over all words in the vocabulary at time-step $i . W_{v}$ and $b_{v}$ are the learning parameters.

\section{Pointer Mechanism}

Some rare words or OOV words such as named-entities are central to the summary, but they prevent models from learning representations for new words when training. It is commonly dealt with the use of an universal "UNK" token for words representation, but resulting in a poor readability for the generated summaries. In summarization tasks, an intuitive way to handle such OOV words is to simply point to their location in the source document. Inspired by [30] and [14], we use a pointer mechanism between the primary encoder and the decoder in our dual encoding model. We allow copying words via pointing, along with generating words from a fixed vocabulary. A soft switch $P_{p}$ is used to choose between generating a word from the fixed vocabulary by sampling from $P_{v}$, and copying a word from the input sequence by sampling from the attention distribution $a_{i} . P_{p}$ is a generation probability for time-step $i$, which is calculated as

$$
P_{p}=\sigma\left(w_{c}^{T} c_{i}+w_{h}^{T} h_{i}^{d}+w_{y}^{T} y_{i}+w_{d}^{T} C^{d}+b_{g}\right),
$$

where $w_{c}, w_{h}, w_{y}, w_{d}$ and $b_{g}$ are the learning parameters. $c_{i}$ is the context vector, $h_{i}^{d}$ is the decoder hidden state, $y_{i}$ is the decoder input and $C^{d}$ is the content representation of partial decoded sequence. $\sigma$ is the sigmoid function, hence, $P_{p} \in[0,1]$.

For each document, we use an extended vocabulary to donate the union of the fixed vocabulary and all words appearing in the source document. The probability distribution over the extended vocabulary is calculated as

$$
P_{w}=P_{p} P_{v}(w)+\left(1-P_{p}\right) \sum_{j: w_{j}=w} a_{i j} .
$$

Note that if $w$ is an OOV word, then $P_{v}(w)$ is zero. Similarly, if $w$ does not appear in the source document, then $\sum_{j: w_{j}=w} a_{i j}$ is also zero. The pointer mechanism is more robust in dealing with rare words as it uses the hidden-state representation of rare words from the encoder to decide which word from the source document to point to. The model is still able to accurately point to unseen words which do not appear in the target vocabulary, because the hidden state depends on the entire context of the word.

During training, the loss for time-step $i$ is the negative $\log$ likelihood of the target word $w_{i}$, i.e., $\mathcal{L}_{i}=-\log P\left(w_{i}\right)$. Therefore, the overall loss for the whole sequence is

$$
\mathcal{L}=\frac{1}{T} \sum_{i=0}^{T} \mathcal{L}_{i}
$$

where $T$ denotes the target sequence length.

\section{E. Repetition Avoidance}

For sequence-to-sequence models, repetition is a common problem in sequence generation tasks, especially notable in generating multi-sentence text. In our dual encoding model, an enhanced mechanism is used to solve this problem. On one hand, the secondary encoder generates an encoding feature vector every $K$ steps, which makes the decoder "remembers" the content produced in the earlier time-steps to avoid the 
repetition. On the other hand, we use the coverage mechanism [14], in which the coverage vector $c^{v}$ is defined to the sum of attention distributions over all previous decoder timesteps:

$$
c_{i}^{v}=\sum_{i^{\prime}=0}^{i-1} a_{i^{\prime}} .
$$

Note that $c_{0}^{v}$ is a zero vector, because none of the source document has been covered on the first decoding time-step. Next, the coverage vector is also used as extra input to the attention mechanism in Eq. (7). Hence, the formula for attention mechanism is updated to

$$
e_{i j}=v_{a}^{T} \tanh \left(W_{a} h_{i-1}^{d}+U_{a} h_{j}^{p}+W_{c h} c_{i}^{v}\right) .
$$

At the same time, an additional coverage loss is defined to penalize repeatedly attending to the same locations. Combining Eq. (13), the primary loss function is rewritten as

$$
\mathcal{L}=\frac{1}{T} \sum_{i=0}^{T}\left(\mathcal{L}_{i}+\lambda \sum_{j} \min \left(a_{i j}, c_{i j}^{v}\right)\right),
$$

where $\lambda$ is a hyper parameter. $i$ and $j$ denote the decoding time-step and the position in input sequence, respectively. The coverage mechanism aims to deal with the repetition problems from the encoder by discouraging the decoder from attending to the same part on the input sequence according to the past attentional weights. Combined the coverage mechanism with the content produced in the earlier time-steps by the decoder, the enhanced mechanism in our work can be regarded as avoiding the repetition from both the encoder and the decoder.

\section{EXPERIMENTAL RESUlTS}

In this section, the datasets for evaluation and the evaluation metric are introduced first, and then the proposed method is compared against with the state-of-the-art methods in the challenging datasets. The experimental results of other stateof-the-art methods are provided from the authors or reproduced from the available source codes. The proposed method is implemented using Tensorflow ${ }^{2}$ It takes about 2 weeks to train our model until the model converges on a machine with a $3.4 \mathrm{GHz}$ Intel $i 7$ processor, $32 \mathrm{~GB}$ memory and a NVIDIA GTX 1080 GPU card with 8 GB memory.

\section{A. Datasets}

For our experiments, we train and test our dual encoding model on the joint CNN/DailyMail dataset, namely a multiple sentences summarization dataset. The dataset is originally constructed for the question answering task [34], and remodified for abstractive summarization task [10]. On average, there are 28 sentences per document in the training set, and an average of $3 \sim 4$ sentences in the reference summaries. Overall, the dataset contains 286,817 in training set, 13,368 in validation set and 11, 487 examples in testing set. Besides, there are on average 781 and 56 tokens in the input articles and the output summaries, respectively.

\footnotetext{
${ }^{2}$ https://www.tensorflow.org/
}

We also use the DUC 2004 corpus as a testing dataset to evaluate our model. It contains 500 documents and their corresponding summaries, where each document has 4 different human-written reference summaries. In our work, we test our dual encoding model on this dataset which is trained on the CNN/DailyMail dataset, and we limit the length of every summary to 30 words since the official evaluation on it is based on limited-length Rouge recall.

\section{B. Experimental Settings}

In our experiments, the batch size is set to 32 when training our model. We set the dimension of hidden states of both encoders and decoder to 256 . We limit the size of vocabulary to 50,000 by selecting the most frequent tokens in the training set. OOV words are represented as token $<\mathrm{UNK}\rangle$. Similar to the settings in the work [14], we do not pretrain the word embeddings, but learn themselves from scratch during training. The dimension of word embeddings are set to 128. The network parameters are randomly initialized over a uniform distribution within $[-0.05,0.05]$, and optimized using Adagrad [39] algorithm. The learning rate and an initial accumulator value is set to 0.15 and 0.1 respectively. We clip gradient with the maximum gradient norm of 5 . In addition, we set the decoding length to 20 for the CNN/DailyMail dataset with long summary, and 10 for the DUC 2004 dataset with relatively short summary.

For speeding up training, we truncate the input sequences to 400 tokens and restrict the length of summaries to 100 tokens on the CNN/DailyMail dataset. At the testing time, we also use the same length settings, and decode the output summaries using beam search with beam size 4 .

\section{Compared Methods}

In this section, we compare the proposed dual encoding model (DEATS) method with the following state-of-the-art methods on the CNN/DailyMail dataset and the DUC 2004 test dataset:

1) DUC 2004 Dataset: We compare the performance of our dual encoding model with the following models on the DUC 2004 dataset.

- TOPIARY [28] using a combination of linguistically motivated compression methods and an unsupervised topic detection algorithm, which is the best performer on the dataset.

- ABS [8] with a local attention-based mechanism to generate each word of the summary conditioned on the input sentence.

- ABS+ [8] combining conventional ABS combined and an additional log-linear extractive summarization model with hand-crafted features.

- RAS-EIman [9] using an attentive encoder and RNN based decoder.

- words-lvt5k-Isent [10] that is an attentional encoderdecoder model with the large vocabulary trick.

- SEASS [40] that is a selective encoding model with a selective gate network to construct a second level sentence representation by controlling the information flow from encoder to decoder. 
2) CNN/DailyMail Dataset: On this joint dataset, we compare the performance of our dual encoding model with the following approaches.

- seq2seq+atten [12] that is a standard sequence-tosequence model with attention mechanism employed for abstractive text summarization.

- words-lvt2k-temp-att [10] that is an abstractive encoderdecoder based model using the temporal attention mechanism from [41] that keeps track of past attentional weights of the decoder and restrains the repetitive parts in the later sequence.

- SummaRuNNer-abs [25] that is a recurrent neural network based sequence model for abstractive summarization and is converted from a extractive model by using a novel training mechanism.

- pointer-generator [14] that is a standard sequenceto-sequence attentional model based hybrid pointergenerator network to deal with rare or OOV words problem.

- pointer-generator+coverage [14] that is improved from "pointer-generator" model by adding a coverage mechanism to discourage the repetition, denoted as "pg+cg" in Table I

- $\mathbf{R L}+\mathbf{M L}$ [17] that is a neural network model with intraattention and a new training approach for abstractive summarization.

Notably, "RL+ML" approach [17] combines maximumlikelihood training and reinforcement training. Likewise, the pointer mechanism, similar to "pointer-generator", is also used in their model. Different from the hybrid training method in their work, all above approaches including ours are the standard supervised sequence prediction model using maximumlikelihood training.

\section{Evaluation}

We evaluate our model using ROUGE metric [42]. ROUGE measures the quality of summary by computing the number of overlapping lexical units. In this paper, we use the scores from Rouge-1, Rouge- 2 and Rouge-L, which respectively measure the matches of unigrams, bigrams and longest common subsequences between the generated summaries and the reference summaries. In our experiments, we randomly select 100 test examples to evaluate the summary quality. The ROUGE scores are obtained using the pyrouge package 3

\section{E. Results on CNN/DailyMail Corpus}

We report the experimental results of various models on the CNN/DailyMail testing set in Table I From the results shown in Table I] our dual encoding model achieves the-state-of-theart performance. This is attributed to the following factors. Firstly, compared with the "pg+cg" approach only using a coverage mechanism to discourage the repetition, "DEATS" uses an enhanced repetition avoid mechanism which combines the coverage mechanism and the previously generated output by decoder to improve the quality of the generated summary.

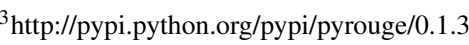

TABLE I

PERFORMANCE COMPARISON OF VARIOUS MODELS ON THE CNN/DAILY MAIL TESTING SET USING ROUGE F1 SCORE.

\begin{tabular}{cccc}
\hline Method & Rouge-1 & Rouge-2 & Rouge-L \\
\hline seq2seq+atten & 31.34 & 11.79 & 28.10 \\
words-lvt2k-temp-att & 35.46 & 13.30 & 32.65 \\
SummaRuNNer-abs & 37.50 & 14.50 & 33.40 \\
pointer-generator & 36.44 & 15.66 & 33.42 \\
RL+ML & 39.87 & 15.82 & 36.90 \\
pg+cg & 39.53 & 17.28 & 36.38 \\
DEATS & $\mathbf{4 0 . 8 5}$ & $\mathbf{1 8 . 0 8}$ & $\mathbf{3 7 . 1 3}$ \\
\hline
\end{tabular}

TABLE II

PERFORMANCE COMPARISON OF VARIOUS MODELS ON THE DUC 2004 TESTING SET USING ROUGE RECALL SCORE.

\begin{tabular}{cccc}
\hline Method & Rouge-1 & Rouge-2 & Rouge-L \\
\hline TOPIARY & 25.12 & 6.46 & 20.12 \\
ABS & 26.55 & 7.06 & 22.05 \\
ABS+ & 28.18 & 8.49 & 23.81 \\
RAS-Elman & 28.97 & 8.26 & 24.06 \\
words-lvt5k-lsent & 28.61 & 9.42 & 25.24 \\
SEASS & 29.21 & 9.56 & 25.51 \\
DEATS & $\mathbf{2 9 . 9 1}$ & $\mathbf{9 . 6 1}$ & $\mathbf{2 5 . 9 5}$ \\
\hline
\end{tabular}

Secondly, the compared methods generate the complete target summary at once and just conduct one encoding process on the input sequence; while our "DEATS" method adopts a dual encoding mechanism and multi-steps decoding operation. Specifically, the secondary encoding in dual encoding mechanism is more likely to fulfil a fine and selective encoding based on the input and the previous output that tends to help decoder produce better summary. In addition, it is observed that ROUGE scores seem close in these methods based on deep learning. The reason is that Rouge scores are really hard to improve too much. Generally, 1 2 Rouge score indicates an important performance improvement.

\section{F. Results on DUC 2004 Corpus}

We also test "DEATS" on the out-of-domain DUC 2004 dataset which is trained on the CNN/DailyMail dataset. Evaluation of our "DEATS" method uses the limited-length Rouge Recall at 75 bytes. According to the results in Table II, our method achieves the best performance. It is worth mentioning that "DEATS" just achieves slightly better performance than "SEASS" (e.g., 29.91 vs 29.21 for Rouge-1). On the other hand, in terms of the CNN/DailyMail dataset with relatively long summary, our method achieves more improvement in performance (e.g., 40.85 vs 39.87 for Rouge- 1 in Table I). The potential reason may be that the dual encoding mechanism in our model is more suitable for long sequence generation tasks while the summary in the DUC 2004 dataset is relatively short.

\section{Discussion}

We further perform experiments to study the effect of different aspects of our "DEATS" method on the performance. We use the CNN/DailyMail dataset to conduct the experiments. 
TABLE III

EXAMPLES GENERATED FROM THE “DEATS” METHOD ON THE CNN/DAILYMAIL TESTING SET UNDER THE DIFFERENT DECODING LENGTHS AND THE ROUGE SCORES CORRESPOND TO THE SPECIFIC EXAMPLE AND $K$ DENOTES THE DECODING LENGTH.

\begin{tabular}{|c|}
\hline rce Document 1: \\
\hline $\begin{array}{l}\text { a duke student has admitted to hanging a noose made of rope from a tree near a student union, university officials said thursday . the prestigious private } \\
\text { school did n't identify the student, citing federal privacy laws . in a news release, it said the student was no longer on campus and will face student conduct } \\
\text { review . the student was identified during an investigation by campus police and the office of student affairs and admitted to placing the noose on the tree early } \\
\text { wednesday, the university said . officials are still trying to determine if other people were involved . criminal investigations into the incident are ongoing as } \\
\text { well . students and faculty members marched wednesday afternoon chanting " we are not afraid . we stand together, " after pictures of the noose were passed } \\
\text { around on social media ....... in the news release, the vice president for student affairs called the noose incident a " cowardly act ." " to whomever committed } \\
\text { this hateful and stupid act, i just want to say that if your intent was to create fear, it will have the opposite effect, " larry moneta said wednesday . duke } \\
\text { university is a private college with about } 15,000 \text { students in durham, north carolina. }\end{array}$ \\
\hline Ground Truth Summary: \\
\hline $\begin{array}{l}\text { student is no longer on duke university campus and will face disciplinary review. school officials identified student during investigation and the person admitted } \\
\text { to hanging the noose, duke says. the noose, made of rope, was discovered on campus about } 2 \text { a.m. }\end{array}$ \\
\hline DEATS, $K=10$ (Rouge-1:50.00, Rouge-2:16.22, Rouge-L:47.37) \\
\hline $\begin{array}{l}\text { duke student has admitted to hanging a noose made of rope from a tree near student union. the student was identified during an investigation by campus police } \\
\text { and the office of student affairs. }\end{array}$ \\
\hline DEATS, $K=20$ (Rouge-1:52.87, Rouge-2:16.47, Rouge-L:48.28) \\
\hline $\begin{array}{l}\text { duke student has admitted to hanging a noose made of rope from a tree near a student union. the student was identified during an investigation by campus } \\
\text { police and the office of student affairs and admitted to placing the noose on the tree early wednesday. }\end{array}$ \\
\hline DEATS, $K=30$ (Rouge-1:47.19, Rouge-2:13.79, Rouge-L:40.45) \\
\hline $\begin{array}{l}\text { duke student has admitted to hanging a noose made of rope from a tree near a student union. the student was identified during an investigation by campus } \\
\text { police and the office of student affairs. the incident is one of several recent racist events to affect college students. }\end{array}$ \\
\hline Source Document 2: \\
\hline $\begin{array}{l}\text { this time, it 's official : russia expects north korean leader kim jong un to visit moscow next month for world war ii anniversary celebrations . north korean } \\
\text { representatives have confirmed that kim will be in the russian capital for may } 9 \text { victory day celebrations, russian presidential aide yuri ushakov said wednesday } \\
\text {, according to russian state-run news agency tass . this would mark kim 's first official foreign trip since inheriting the leadership of north korea in late } 2011 \\
\text {. kim will meet with russian president vladimir putin as part of the may visit, tass reported . kim 's trip has been anticipated since late december, when } \\
\text { russian state media reported that moscow had extended an invitation to pyongyang. ...... this year 's victory day marks the } 70 \text { th anniversary of the soviet union } \\
\text { 's victory over nazi germany in world war ii . russia has said it has invited more than } 60 \text { world leaders to the celebrations . kim expected to visit moscow as } \\
\text { north korea , russia foster warmer relations . cnn 's madison park and alla eshchenko contributed to this report. }\end{array}$ \\
\hline Ground Truth Summary: \\
\hline $\begin{array}{l}\text { a russian presidential aide says kim will be in moscow for may } 9 \text { victory day celebrations, news agency reports. this victory day marks the } 70 \text { years since the } \\
\text { soviet victory over germany in world war ii. }\end{array}$ \\
\hline DEATS, $K=10$ (Rouge-1:43.84, Rouge-2:28.17, Rouge-L:38.36) \\
\hline $\begin{array}{l}\text { russia expects north korean leader kim jong un to visit moscow next month for world war ii anniversary. north korean representatives have confirmed that kim } \\
\text { will be in the russian capital for may } 9 \text { victory day celebrations. }\end{array}$ \\
\hline DEATS, $K=20$ (Rouge-1:43.24, Rouge-2:27.78, Rouge-L:37.84) \\
\hline $\begin{array}{l}\text { russia expects north korean leader kim jong un to visit moscow next month for world war ii anniversary celebrations. north korean representatives have confirmed } \\
\text { that kim will be in the russian capital for may } 9 \text { victory day celebrations. }\end{array}$ \\
\hline DEATS, $K=30$ (Rouge-1:38.64, Rouge-2:23.26, Rouge-L:34.09) \\
\hline $\begin{array}{l}\text { russia expects north korean leader kim jong un to visit moscow next month for world war ii anniversary celebrations. north korean representatives have confirmed } \\
\text { that kim will be in the russian capital for may } 9 \text { victory day celebrations. kim will meet with russian president vladimir putin as part of the may visit. }\end{array}$ \\
\hline Source Document 3: \\
\hline $\begin{array}{l}\text { a 32-year-old massachusetts man is facing murder charges, authorities said wednesday, four days after another man 's remains were found in a duffel bag . the } \\
\text { middlesex district attorney 's office said that carlos colina, } 32, \text { will be arraigned the morning of april } 14 \text { for murder in connection with the remains discovered } \\
\text { saturday in cambridge . earlier this week, colina was arraigned on charges of assault and battery causing serious bodily injury and improper disposal of a body } \\
\text {. a middlesex county judge then revoked bail for colina in another case he 's involved in , for alleged assault and battery . the victim in that case is different } \\
\text { from the one whose remains were found in recent days . police were notified saturday morning about a suspicious item along a walkway in cambridge. ...... } \\
\text { that location is near the cambridge police department headquarters . the remains at both locations belonged to the same victim, identified monday as jonathan } \\
\text { camilien, } 26 \text {. camilien and colina knew each other, according to authorities . "this was a gruesome discovery, " district attorney marian ryan said . cnn 's } \\
\text { kevin conlon contributed to this report. }\end{array}$ \\
\hline Ground Truth Summary: \\
\hline $\begin{array}{l}\text { prosecutor : carlos colina , } 32 \text {, will be arraigned on the murder charge next week. he 's already been arraigned for alleged assault and battery, improper } \\
\text { disposal of a body. body parts were found in a duffel bag and a common area of an apartment building. }\end{array}$ \\
\hline DEATS, $K=10$ (Rouge-1:60.27, Rouge-2:42.25, Rouge-L:54.80) \\
\hline $\begin{array}{l}\text { carlos colina , 32, will be arraigned for murder charge on the morning of april 14. colina was arraigned for alleged assault and battery and improper disposal } \\
\text { of a body. }\end{array}$ \\
\hline DEATS, $K=20$ (Rouge-1:59.77, Rouge-2:42.35, Rouge-L:55.17) \\
\hline $\begin{array}{l}\text { carlos colina , } 32, \text { will be arraigned for murder charge on the morning of april } 14 \text {. colina was arraigned for alleged assault and battery and improper disposal } \\
\text { of a body. the victim is different from the one whose remains were found in recent days . }\end{array}$ \\
\hline DEATS, $K=30$ (Rouge-1:58.54, Rouge-2:40.00, Rouge-L:53.66) \\
\hline $\begin{array}{l}\text { carlos colina , } 32 \text {, will be arraigned for murder charge on the morning of april } 14 \text {. colina was arraigned for alleged assault and battery and improper di } \\
\text { of a body. a middlesex county judge then revoked bail for colina. }\end{array}$ \\
\hline
\end{tabular}

\section{A. Influence of Decoding Length}

In our dual encoding model, we conduct a multi-step secondary decoding process for one iteration. To evaluate the influence of different decoding lengths on the performance, we set the decoding length $K=\{10,15,20,25,30,40,50,100\}$. When the decoding length is set to 100 , it means that we decode the whole output sequence at one time.

As shown in Fig. 2, we can see that higher precision and lower recall are obtained when the decoding length is set to a smaller value, while setting a larger value results in the opposite result. We can obtain a good trade-off between recall and precision when the decoding length is set to $20 \sim 25$. In this work, we fix the decoding length as 20 when decoding this dataset. Selecting a variable decoding length automatically may improve our model performance further, but it is a discrete action and needs to incorporate the reinforcement learning method in the model training.

We also report the ROUGE F1 score for our "DEATS" under different decoding lengths in Table IV] on this dataset. From the Table IV, we can see that the performance decreases 


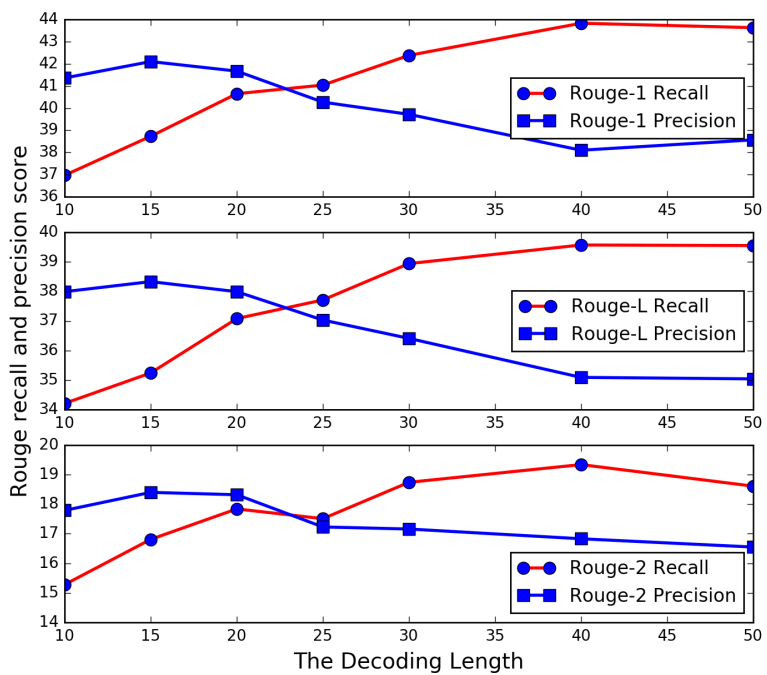

Fig. 2. The Rouge recall and precision scores on the CNN/DailyMail testing set.

notably for too small decoding length, and we obtain better results when the decoding length is set to $20 \sim 40$. That is because smaller decoding length gives rise to the increase of precision and the decrease of recall in ROUGE metric while bigger decoding length results in the opposite result. We need to balance recall and precision by setting a proper decoding length. In Table III] we show the examples generated by our "DEATS" approach based on the different decoding lengths. To summarize, too large decoding length makes the secondary encoder out of function, while too small decoding length is not able to capture enough information and increases computational cost due to more secondary encoding operation.

Notably, the first two examples in Table III show the generated summaries are directly extracted from the source documents. This is because the words in source text are more likely generated with higher probability. However, some words not existing in source text are still able to be generated, such as the third example in Table III. The same phenomenon is also found in two other classic abstractive text summarization methods [14], [17].

TABLE IV

PERFORMANCE COMPARISON OF OUR DUAL ENCODING MODELS FOR DIFFERENT DECODING LENGTHS ON THE CNN/DAILY MAIL TESTING SET USING ROUGE F1 SCORE.

\begin{tabular}{cccc}
\hline DEATS variants & Rouge-1 & Rouge-2 & Rouge-L \\
\hline DEATS-10 & 38.26 & 16.44 & 34.90 \\
DEATS-15 & 40.35 & 17.57 & 36.72 \\
DEATS-20 & $\mathbf{4 0 . 8 5}$ & 18.08 & 37.13 \\
DEATS-25 & 40.62 & 17.37 & $\mathbf{3 7 . 3 7}$ \\
DEATS-30 & 40.71 & $\mathbf{1 8 . 1 8}$ & 37.23 \\
DEATS-40 & 40.76 & 17.99 & 37.20 \\
DEATS-50 & 40.75 & 17.52 & 37.16 \\
DEATS-100 & 39.85 & 17.45 & 36.58 \\
\hline
\end{tabular}

\section{B. Effectiveness of Dual Encoding Mechanism}

We investigate the influence of different modules in our method in Table $\mathrm{V}$. Specifically, we remove one or two of the three modules (i.e., "PM" short for the pointer mechanism, "DEM" short for the dual encoding mechanism and "RAM" short for the repetition avoidance mechanism) from "DEATS" each time. Notably, Our dual encoding model without the dual encoding mechanism and all three modules degenerates into "pg+cg" model and "seq2seq+atten" model in Table I respectively. It degenerates into "pointer-generator" model when without the dual encoding mechanism and the repetition avoidance mechanism.

As presented in Table V, "DEATS" considering all the components shows a significant improvement of the performance over its variants. If the repetition avoidance mechanism (i.e., RAM) is excluded, the Rouge scores decrease the most, indicating that the repetition phenomenon affects the performance of the generated summaries, and "RAM" in our model is able to restrain the repetition phenomenon well. Without the dual encoding mechanism (i.e., "DEM"), Rouge-1, Rouge-2 and Rouge- $\mathrm{L}$ are reduced by $1.32,0.80$ and 0.75 , respectively, which is still a big decrease in summarization tasks. This indicates the secondary encoder in our model conducts a more fine encoding to help the model consider richer and more accurate information. If there is no the pointer mechanism (i.e., "PM"), the performance is also degraded. That is because some OOV words are more easier to appear in the generated summaries. Using all the three components is critical to the performance of "DEATS".

TABLE V

PERFoRMANCE COMPARISON OF DEATS VARIANTS ON THE CNN/DAILY MAIL TESTING SET USING ROUGE F1 SCORE.

\begin{tabular}{cccc}
\hline DEATS variants & Rouge-1 & Rouge-2 & Rouge-L \\
\hline DEATS & $\mathbf{4 0 . 8 5}$ & $\mathbf{1 8 . 0 8}$ & $\mathbf{3 7 . 1 3}$ \\
\hline DEATS_w/o_RAM & 38.05 & 16.16 & 34.32 \\
DEATS_w/o_DEM & 39.53 & 17.28 & 36.38 \\
DEATS_w/o_PM & 39.61 & 17.12 & 36.45 \\
DEATS_w/o_PM+RAM & 32.63 & 12.97 & 29.23 \\
DEATS_w/o_DEM+RAM & 36.44 & 15.66 & 33.42 \\
DEATS_w/o_ALL & 31.34 & 11.79 & 28.10 \\
\hline
\end{tabular}

\section{Effectiveness of Repetition Avoidance Mechanism}

Our dual encoding model uses an enhanced repetition avoidance mechanism which combines the existing coverage mechanism with the already produced output by the decoder. To verify the capability of eliminating the repetition phenomenon in the generated summaries without coverage mechanism, we set the decoding length to a smaller value to make the decoder better "remember" the decoded information in the earlier timesteps. We show some examples generated by our "DEATS" approach without using coverage mechanism in Table VI The results indicate that our dual encoding model without coverage mechanism is still capable of dampening the repetition when the decoding length is set to a small value. 
TABLE VI

EXAMPLES FROM THE CNN/DAILY MAIL TESTING DATASET GENERATED BY OUR “DEATS" APPROACH.

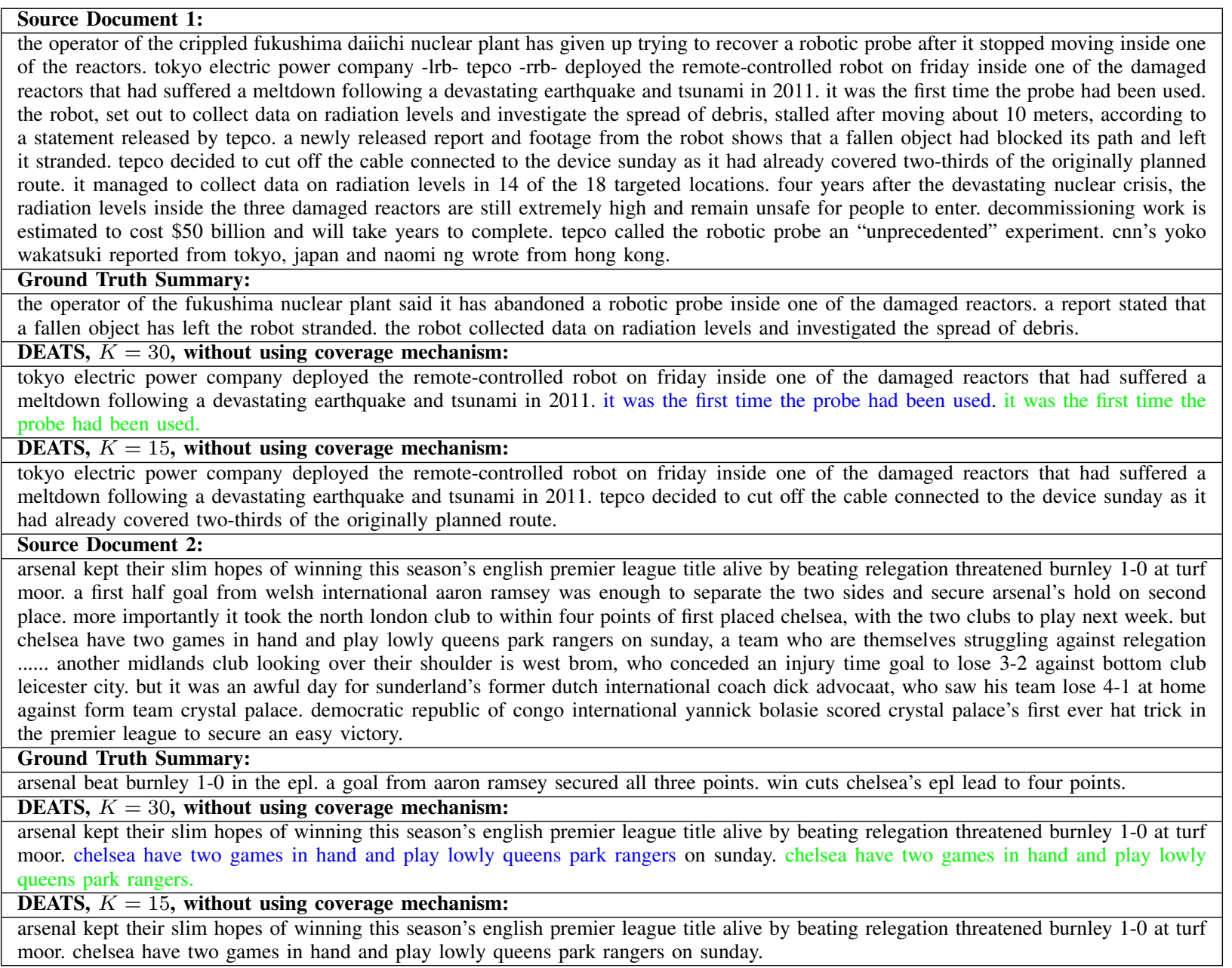

\section{Importance Weight Visualization}

The secondary encoder in our model reads each word in input sequence in the form of skip-connections as shown in Fig. 1. This makes the secondary encoder different from the ordinary encoder. Our secondary encoder will read the input sequence more than once in one training iteration. That is to say, it conducts a secondary encoding every $K$ decoding steps based on the input text and the previous output. Moreover, every secondary encoding will pay attention to the different part of the input sequence. We model the importance of each word as $\alpha_{t}$. Therefore, we can visualize the importance weight of each word to observe whether the secondary encoder assigns different weights to different words in every secondary encoding. As shown in Fig. 3, the secondary encoding is shown for three times and it pays attention to the different part every time. Specifically, the encoder assigns different weights to each word in the input sequence at one same encoding. Meanwhile, the encoder also assigns different weights to the same words at every different encoding. Notably, for clarity, we only show the importance weight visualization for each secondary encoding on the partial input sequence.

\section{CONCLUSION}

In this paper, we present a dual encoding model which extends the sequence-to-sequence framework for abstractive text summarization. Our model is built on a basic encoderdecoder model with attention mechanism, the pointer mechanism and the repetition avoidance mechanism. Different from the standard encoder-decoder model, the dual encoding model decodes the whole output sequence by stages and produces the partial fixed-length sequence at each stage. A combination of the dual encoding mechanism and the basic approaches could make them benefit from each other. The extensive experiments on the CNN/DailyMail and DUC 2004 datsets show that our dual encoding model achieves the state-of-the-art results compared to existing methods.

In our future work, we plan to focus on how to balance precision and recall to further boost F1 performance by selecting dynamic decoding length automatically based on reinforcement learning. Meanwhile, in order to achieve better performance, we also attempt to train our dual encoding model by using a hybrid training objective with reinforcement learning training and maximum-likelihood training. 


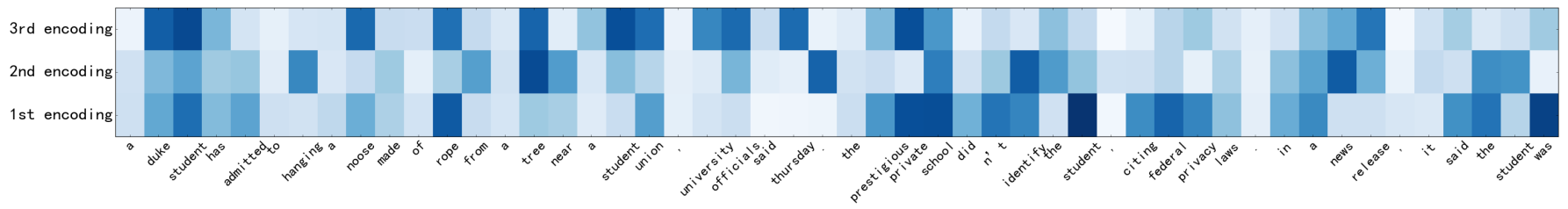

Fig. 3. Importance weight visualization on the partial input text.

\section{ACKNOWLEDGMENT}

The authors would like to thank Zhaoqing Peng and Fei Zhang for helpful discussions about model training and network parameters tuning tricks. They would also like to thank the anonymous reviewers for their help in improving this paper.

\section{REFERENCES}

[1] R. Collobert, J. Weston, L. Bottou, M. Karlen, K. Kavukcuoglu, and P. P. Kuksa, "Natural language processing (almost) from scratch," Journal of Machine Learning Research, vol. 12, pp. 2493-2537, 2011.

[2] K. Cho, B. van Merrienboer, Ç. Gülçehre, D. Bahdanau, F. Bougares, H. Schwenk, and Y. Bengio, "Learning phrase representations using RNN encoder-decoder for statistical machine translation," in Proceedings of the 2014 Conference on Empirical Methods in Natural Language Processing, EMNLP 2014, October 25-29, 2014, Doha, Qatar, A meeting of SIGDAT, a Special Interest Group of the ACL, 2014, pp. 1724-1734.

[3] I. Sutskever, O. Vinyals, and Q. V. Le, "Sequence to sequence learning with neural networks," in Advances in Neural Information Processing Systems 27: Annual Conference on Neural Information Processing Systems 2014, December 8-13 2014, Montreal, Quebec, Canada, 2014, pp. 3104-3112.

[4] D. Bahdanau, J. Chorowski, D. Serdyuk, P. Brakel, and Y. Bengio, "End-to-end attention-based large vocabulary speech recognition," in 2016 IEEE International Conference on Acoustics, Speech and Signal Processing, ICASSP 2016, Shanghai, China, March 20-25, 2016, 2016, pp. 4945-4949.

[5] G. Biagetti, P. Crippa, L. Falaschetti, S. Orcioni, and C. Turchetti, "An investigation on the accuracy of truncated dklt representation for speaker identification with short sequences of speech frames," IEEE Transactions on Cybernetics, vol. 47, no. 12, pp. 4235-4249, 2017.

[6] O. Vinyals, A. Toshev, S. Bengio, and D. Erhan, "Show and tell: A neural image caption generator," in IEEE Conference on Computer Vision and Pattern Recognition, CVPR 2015, Boston, MA, USA, June 7-12, 2015, 2015, pp. 3156-3164.

[7] O. Vinyals and Q. V. Le, "A neural conversational model," CoRR, vol. abs/1506.05869, 2015.

[8] A. M. Rush, S. Chopra, and J. Weston, "A neural attention model for abstractive sentence summarization," in Proceedings of the 2015 Conference on Empirical Methods in Natural Language Processing, EMNLP 2015, Lisbon, Portugal, September 17-21, 2015, 2015, pp. 379389.

[9] S. Chopra, M. Auli, and A. M. Rush, "Abstractive sentence summarization with attentive recurrent neural networks," in NAACL HLT 2016, The 2016 Conference of the North American Chapter of the Association for Computational Linguistics: Human Language Technologies, San Diego California, USA, June 12-17, 2016, 2016, pp. 93-98.

[10] R. Nallapati, B. Zhou, C. N. dos Santos, Ç. Gülçehre, and B. Xiang, "Abstractive text summarization using sequence-to-sequence rnns and beyond," in Proceedings of the 20th SIGNLL Conference on Computational Natural Language Learning, CoNLL 2016, Berlin, Germany, August 11-12, 2016, 2016, pp. 280-290.

[11] K. Filippova, E. Alfonseca, C. A. Colmenares, L. Kaiser, and O. Vinyals, "Sentence compression by deletion with lstms," in Conference on Empirical Methods in Natural Language Processing, 2015, pp. 360368.

[12] D. Bahdanau, K. Cho, and Y. Bengio, "Neural machine translation by jointly learning to align and translate," CoRR, vol. abs/1409.0473, 2014.

[13] Ç. Gülçehre, S. Ahn, R. Nallapati, B. Zhou, and Y. Bengio, "Pointing the unknown words," in Proceedings of the 54th Annual Meeting of the Association for Computational Linguistics, ACL 2016, August 7-12, 2016, Berlin, Germany, Volume 1: Long Papers, 2016.
[14] A. See, P. J. Liu, and C. D. Manning, "Get to the point: Summarization with pointer-generator networks," in Proceedings of the 55th Annual Meeting of the Association for Computational Linguistics, ACL 2017, Vancouver, Canada, July 30 - August 4, Volume 1: Long Papers, 2017, pp. 1073-1083.

[15] J. Gu, Z. Lu, H. Li, and V. O. K. Li, "Incorporating copying mechanism in sequence-to-sequence learning," in Proceedings of the 54th Annual Meeting of the Association for Computational Linguistics, ACL 2016, August 7-12, 2016, Berlin, Germany, Volume 1: Long Papers, 2016.

[16] W. Zeng, W. Luo, S. Fidler, and R. Urtasun, "Efficient summarization with read-again and copy mechanism," CoRR, vol. abs/1611.03382, 2016.

[17] R. Paulus, C. Xiong, and R. Socher, "A deep reinforced model for abstractive summarization," CoRR, vol. abs/1705.04304, 2017.

[18] J. L. Neto, A. A. Freitas, and C. A. A. Kaestner, "Automatic text summarization using a machine learning approach," in Advances in Artificial Intelligence, 16th Brazilian Symposium on Artificial Intelligence, SBIA 2002, Porto de Galinhas/Recife, Brazil, November 11-14, 2002, Proceedings, 2002, pp. 205-215.

[19] G. Erkan and D. R. Radev, "Lexrank: Graph-based lexical centrality as salience in text summarization," CoRR, vol. abs/1109.2128, 2011.

[20] K. Wong, M. Wu, and W. Li, "Extractive summarization using supervised and semi-supervised learning," in COLING 2008, 22nd International Conference on Computational Linguistics, Proceedings of the Conference, 18-22 August 2008, Manchester, UK, 2008, pp. 985-992.

[21] K. Filippova and Y. Altun, "Overcoming the lack of parallel data in sentence compression," in Proceedings of the 2013 Conference on Empirical Methods in Natural Language Processing, EMNLP 2013, 18 21 October 2013, Grand Hyatt Seattle, Seattle, Washington, USA, A meeting of SIGDAT, a Special Interest Group of the ACL, 2013, pp. 1481-1491.

[22] T. Mikolov, I. Sutskever, K. Chen, G. S. Corrado, and J. Dean, "Distributed representations of words and phrases and their compositionality," in Advances in Neural Information Processing Systems 26: 27th Annual Conference on Neural Information Processing Systems 2013. Proceedings of a meeting held December 5-8, 2013, Lake Tahoe, Nevada, United States., 2013, pp. 3111-3119.

[23] C. A. Colmenares, M. Litvak, A. Mantrach, and F. Silvestri, "HEADS: headline generation as sequence prediction using an abstract featurerich space," in NAACL HLT 2015, The 2015 Conference of the North American Chapter of the Association for Computational Linguistics: Human Language Technologies, Denver, Colorado, USA, May 31 - June 5, 2015, 2015, pp. 133-142.

[24] J. Cheng and M. Lapata, "Neural summarization by extracting sentences and words," in Proceedings of the 54th Annual Meeting of the Association for Computational Linguistics, ACL 2016, August 7-12, 2016, Berlin, Germany, Volume 1: Long Papers, 2016.

[25] R. Nallapati, F. Zhai, and B. Zhou, "Summarunner: A recurrent neural network based sequence model for extractive summarization of documents," in Proceedings of the Thirty-First AAAI Conference on Artificial Intelligence, February 4-9, 2017, San Francisco, California, USA., 2017, pp. 3075-3081.

[26] Z. Yong, J. E. Meng, Z. Rui, and M. Pratama, "Multiview convolutional neural networks for multidocument extractive summarization," IEEE Transactions on Cybernetics, vol. PP, no. 99, pp. 1-13, 2016.

[27] P. Over, H. Dang, and D. Harman, "DUC in context," Inf. Process. Manage., vol. 43, no. 6, pp. 1506-1520, 2007.

[28] D. Zajic, B. Dorr, and R. Schwartz, "Bbn/umd at duc-2004: Topiary," in Document Understanding Conference at Nlt/naacl, 2004, pp. 112-119.

[29] B. Hu, Q. Chen, and F. Zhu, "LCSTS: A large scale chinese short text summarization dataset," in Proceedings of the 2015 Conference on Empirical Methods in Natural Language Processing, EMNLP 2015, Lisbon, Portugal, September 17-21, 2015, 2015, pp. 1967-1972. 
[30] O. Vinyals, M. Fortunato, and N. Jaitly, "Pointer networks," in Advances in Neural Information Processing Systems 28: Annual Conference on Neural Information Processing Systems 2015, December 7-12, 2015, Montreal, Quebec, Canada, 2015, pp. 2692-2700.

[31] Z. Tu, Z. Lu, Y. Liu, X. Liu, and H. Li, "Modeling coverage for neural machine translation," in Proceedings of the 54th Annual Meeting of the Association for Computational Linguistics, ACL 2016, August 7-12, 2016, Berlin, Germany, Volume 1: Long Papers, 2016.

[32] H. Mi, B. Sankaran, Z. Wang, and A. Ittycheriah, "Coverage embedding models for neural machine translation," in Proceedings of the 2016 Conference on Empirical Methods in Natural Language Processing, EMNLP 2016, Austin, Texas, USA, November 1-4, 2016, 2016, pp. $955-$ 960.

[33] Q. Chen, X. Zhu, Z. Ling, S. Wei, and H. Jiang, "Distraction-based neural networks for document summarization," CoRR, vol. abs/1610.08462, 2016.

[34] K. M. Hermann, T. Kociský, E. Grefenstette, L. Espeholt, W. Kay, M. Suleyman, and P. Blunsom, "Teaching machines to read and comprehend," in Advances in Neural Information Processing Systems 28. Annual Conference on Neural Information Processing Systems 2015, December 7-12, 2015, Montreal, Quebec, Canada, 2015, pp. 1693-1701.

[35] R. Nallapati, F. Zhai, and B. Zhou, "Summarunner: A recurrent neural network based sequence model for extractive summarization of documents," 2016

[36] K. Cho, B. van Merrienboer, C.. Gülçehre, D. Bahdanau, F. Bougares, H. Schwenk, and Y. Bengio, "Learning phrase representations using RNN encoder-decoder for statistical machine translation," in Proceedings of the 2014 Conference on Empirical Methods in Natural Language Processing, EMNLP 2014, October 25-29, 2014, Doha, Qatar, A meeting of SIGDAT, a Special Interest Group of the ACL, 2014, pp. 1724-1734.

[37] A. Graves, Supervised Sequence Labelling with Recurrent Neural Networks, ser. Studies in Computational Intelligence. Springer, 2012, vol. 385 .

[38] J. Chung, C. Gülçehre, K. Cho, and Y. Bengio, "Empirical evaluation of gated recurrent neural networks on sequence modeling," CoRR, vol. abs/1412.3555, 2014

[39] J. C. Duchi, E. Hazan, and Y. Singer, "Adaptive subgradient methods for online learning and stochastic optimization," Journal of Machine Learning Research, vol. 12, pp. 2121-2159, 2011.

[40] Q. Zhou, N. Yang, F. Wei, and M. Zhou, "Selective encoding for abstractive sentence summarization," in Meeting of the Association for Computational Linguistics, 2017, pp. 1095-1104.

[41] B. Sankaran, H. Mi, Y. Al-Onaizan, and A. Ittycheriah, "Temporal attention model for neural machine translation," CoRR, vol. abs/1608.02927, 2016.

[42] C. Flick, "Rouge: A package for automatic evaluation of summaries," in The Workshop on Text Summarization Branches Out, 2004, p. 10.

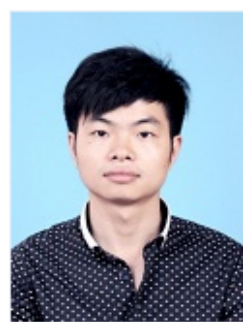

Kaichun Yao is a Ph.D. candidate in School of Computer and control Engineering, University of Chinese Academy of Sciences, Beijing, China. His research interests include natural language processing, knowledge graph, deep learning and reinforcement learning.

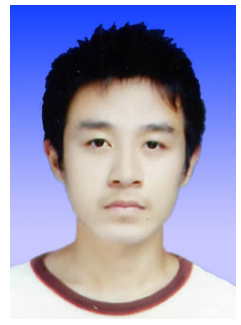

Libo Zhang received the bachelor's degree from Anhui University, the master's degree from University of Electronic Science and Technology of China, and the doctor's degree from University of Chinese Academy of Science. Now $\mathrm{He}$ is an assistant professor in Institute of Software, Chinese Academy of Sciences, Beijing, China. His research interests include image processing, pattern recognition, knowledge graph and deep learning.

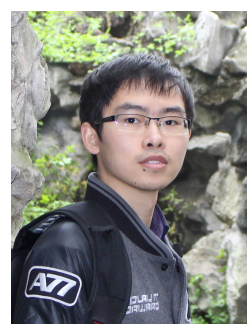

Dawei Du received the B.Eng. degree in automation and the M.S. degree in detection technology and automatic engineering from the University of Electronic Science and Technology of China, Chengdu, China, in 2010 and 2013, respectively. He is currently pursuing the Ph.D. degree with the School of Computer and Control Engineering, University of Chinese Academy of Sciences, Beijing, China. His current research interests include visual tracking, video segmentation and deep learning.

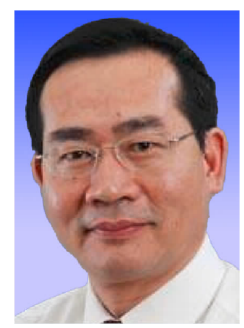

Tiejian Luo is currently a professor in School of Computer and control Engineering, University of Chinese Academy of Sciences (UCAS), Beijing, China. He is also the Director of the Information Dynamic and Engineering Applications Laboratory in UCAS. His main research topics include web mining, large scale web performance optimization and distributed storage systems.

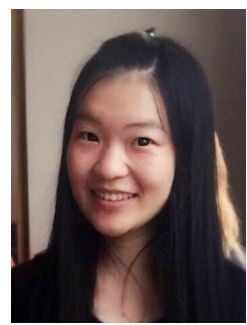

Lili Tao is a Senior Lecturer at the University of the West of England, UK. She is also an Honorary Researcher at the University of Bristol, UK. Her research area is computer vision and robotics. She is particularly interested in developing methods for estimating and analysing deformable and articulated objects, such as human motion modelling and analysis, physical activity monitoring and facial articulated assessment.

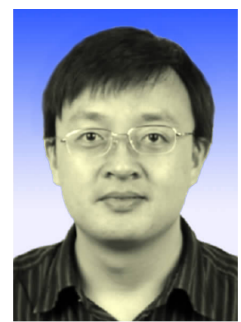

Yanjun Wu received a Ph.D. degree from the Institute of Software, Chinese Academy of Sciences (ISCAS). Currently he is a research professor at ISCAS and his research interests include operating systems and system security. 